\title{
Siglen und Abkürzungen für Ausgaben und wissenschaftliche Literatur
}

$A A D u W$

AA Epen

Adelung

$A S$

Aus Herders Nachlaß

$B G$
Goethe: Aus meinem Leben. Dichtung und Wahrheit. Historisch-kritische Ausgabe bearbeitet von Siegfried Scheibe (Akademie-Ausgabe). Bd 1: Text. Berlin 1970. Bd 2: Überlieferung, Variantenverzeichnis und Paralipomena. Berlin 1974.

Goethe. Epen. Historisch-kritische Ausgabe bearbeitet von Siegfried Scheibe (Akademie-Ausgabe). Bd 1: Text. Berlin 1958. Bd 2: Überlieferung, Varianten und Paralipomena. Berlin 1963.

Johann Christoph Adelung: Grammatisch-kritisches Wörterbuch der Hochdeutschen Mundart, mit beständiger Vergleichung der übrigen Mundarten, besonders aber der Oberdeutschen. Zweyte vermehrte und verbesserte Ausgabe. 4 Tle. Leipzig 1793-1801.

Goethes Amtliche Schriften. Veröffentlichung des Staatsarchivs Weimar. Hrsg. von Willy Flach. 4 Bde. Weimar 1950-1987. - Bd 1: Goethes Tätigkeit im Geheimen Consilium. T. 1: Die Schriften der Jahre 1776-1786. Bearbeitet von Willy Flach. Weimar 1950. - Bd 2: Goethes Tätigkeit im Geheimen Consilium. Seine Schriften der Jahre 1788-1819. Bearbeitet von Helma Dahl. 1. Halbbd: 1788 1797. Weimar 1968; 2. Halbbd: 1798-1819. Weimar 1970. - Bd 3: Goethes Tätigkeit im Geheimen Consilium. Erläuterungen zu den Schriften der Jahre 1788-1819. Bearbeitet von Helma Dahl. Weimar 1972. - Bd 4: Goethes Tätigkeit im Geheimen Consilium. Register. Bearbeitet von Helma Dahl. Weimar 1987.

Aus Herders Nachlaß. Ungedruckte Briefe von Herder und dessen Gattin, Goethe, Schiller, Klopstock, Lenz, Jean Paul, Claudius, Lavater, Jacobi und andern bedeutenden Zeitgenossen. Hrsg. von Heinrich Düntzer und Ferdinand Gottfried von Herder. 3 Bde. Frankfurt a. M. 1856-1857.

Goethe: Begegnungen und Gespräche. Bd 1-2. Hrsg. von Ernst Grumach und Renate Grumach. Berlin 
Biedermann, Unger Briefe

Blumenbach, Correspondence

Bobé, Reventlowske

Familiekreds

Bojanowski, Carl August

Bothe, Residenzschloß

Böttiger, Literarische Zustände ${ }^{1}$
1965-1966. - Bd 3-6. Begründet von Ernst Grumach und Renate Grumach. Hrsg. von Renate Grumach. Berlin, New York 1977-1999. - Bd 8: Begründet von Ernst Grumach und Renate Grumach. Hrsg. von Renate Grumach. Bearb. von Anke Schmidt-Peter. Berlin, New York 2013. Bd 10: Begründet von Ernst Grumach und Renate Grumach. Hrsg. von Renate Grumach und Bastian Röther. Bearb. von Angelika Reimann. Berlin, New York 2018. Bd 14: Begründet von Ernst Grumach und Renate Grumach. Hrsg. von Renate Grumach. Bearb. von Angelika Reimann. Berlin, New York 2011. - Bd 3: 1786-1792 (1977). - Bd 4: 1793-1799 (1980). Johann Friedrich Unger im Verkehr mit Goethe und Schiller. Briefe und Nachrichten. Mit einer einleitenden Übersicht über Ungers Verlegertätigkeit von Flodoard Freiherrn von Biedermann. Berlin 1927.

Dougherty, Frank William Peter: The correspondence of Friedrich Wilhelm Blumenbach. Revised, augmented an edited by Norbert Klatt. Vol. 1-5, Göttingen 2006-2013. - Vol. 4: 1791-1795. Letters 645-945 (Brosamen zur BlumenbachForschung. Begründet und hrsg. von Norbert Klatt. Der Reihe fünfter Band). Göttingen 2012 (Online-Edition).

Efterladte Papirer fra den Reventlowske Familiekreds i tidsrummet 1770-1827. 10 Bind. Udgivne $\langle\ldots\rangle$ ved Louis Bobé. Kjøbenhavn 1895-1931. Bind 3: Grevinde Louise Stolbergs Breve. I Udvalg. Samt Breve fra Greverne Christion og Frederik Leopold Stolberg. Kjøbenhavn 1896.

P〈aul〉 von Bojanowski: Carl August als Chef des 6. Preuß. Kürassier-Regiments 1787-1794. Weimar 1894.

Rolf Bothe: Dichter, Fürst und Architekten. Das Weimarer Residenzschloß vom Mittelalter bis zum Anfang des 19. Jahrhunderts. Ostfildern-Ruit 2000. Literarische Zustände und Zeitgenossen. In Schilderungen aus Karl Aug. Böttiger's handschriftlichem Nachlasse. Hrsg. von K. W. Böttiger, Hofrathe und Professor zu Erlangen. 2 Bde. Leipzig 1838. 
Böttiger, Literarische Zustände ${ }^{2}$

Brachvogel, Theater Berlin

Burkhardt, Repertoire

Büsch,

Handlungs-Akademie

Callot bis Greuze

Carl August-Anna Amalia

Carl August-Goethe

Carl August-Goethe ${ }^{2}$

Charlotte von Schiller
Karl August Böttiger: Literarische Zustände und Zeitgenossen. Begegnungen und Gespräche im klassischen Weimar. Hrsg. von Klaus Gerlach und René Sternke. Berlin 1998.

A〈lbert $\rangle\langle$ mil $\rangle$ Brachvogel: Geschichte des Königlichen Theaters zu Berlin nach Archivalien des Königl. Geh. Staats-Archivs und des Königlichen Theaters. 2 Bde. Berlin 1877/78. - Bd 2: Die Königliche Oper unter Freiherrn von der Reck und das National-Theater bis zu Iffland. Ein Beitrag zur Geschichte Berlins und des deutschen Theaters. Nach Originalquellen. 1878.

Das Repertoire des Weimarischen Theaters unter Goethes Leitung 1791-1817. Bearbeitet und hrsg. von Dr. C〈arl $\rangle\langle$ ugust $\rangle\langle\langle u g o\rangle$ Burkhardt. Hamburg und Leipzig 1891 (Theatergeschichtliche Forschungen. Hrsg. von Berthold Litzmann. I).

Die Hamburgische Handlungs-Akademie. Johann Georg Büsch. Hrsg. von Klaus Friedrich Pott und Jürgen Zabeck. Paderborn 2001 (Wirtschaftspädagogisches Forum hrsg. von Dieter Erler und Peter F. E. Sloane. Bd 17).

David Mandrella, Hermann Mildenberger, Benjamin Peronnet, Pierre Rosenberg: Von Callot bis Greuze. Französische Zeichnungen des 17. und 18. Jahrhunderts (Im Blickfeld der Goethezeit. Bd V). Berlin 2005.

Briefe des Herzogs Carl August von SachsenWeimar an seine Mutter die Herzogin Anna Amalia. Oktober 1774 bis Januar 1807. Hrsg. von Alfred Bergmann (Jenaer Germanistische Forschungen. Bd 30. Hrsg. von A〈lfred Leitzmann). Jena 1938.

Briefwechsel des Großherzogs Carl August von Sachsen-Weimar-Eisenach mit Goethe in den Jahren von 1775 bis 1828. Hrsg. von Carl Vogel. 2 Bde. Weimar 1863.

Briefwechsel des Herzogs-Großherzogs Carl August mit Goethe. Hrsg. von Hans Wahl. 3 Bde. Berlin 1915-1918.

Charlotte von Schiller und ihre Freunde. Hrsg. von Ludwig Urlichs. 3 Bde. Stuttgart 1860, 1862, 1865. 
Corpus

Düntzer, Knebels

Nachlaß

Eigenmann, Hamburger

Theater

Ereignis Weimar
Goethes Sammlungen zur Kunst, Literatur und Naturwissenschaft. Corpus der Goethezeichnungen. Bearbeiter der Ausgabe: Gerhard Femmel. 7 Bde in 10 Teilen. Leipzig 1958-1973. - Bd I. Nr 1-318: Von den Anfängen bis zur italienischen Reise 1786 (1958); Bd II. Nr 1-416: Italienische Reise 1786 bis 1788. Die Landschaften (1960); Bd III. Nr 1-271: Italienische Reise 1786 bis 1788. Antikenund Anatomiestudien. Architektur und Perspektive (1965); Bd IVa. Nr 1-348: Nachitalienische Landschaften (1966); Bd IVb. Nr 1-271: Nachitalienische Zeichnungen 1788 bis 1829. Antike. Porträt. Figurales. Architektur. Theater (1968); Bd Va. Nr 1-390: Die Zeichnungen zur Farbenlehre. Bearbeiter der Ausgabe: Rupprecht Matthaei (1963); Bd Vb. Nr 1-264: Die naturwissenschaftlichen Zeichnungen mit Ausnahme der Farbenlehre. Bestände der Nationalen Forschungs- und Gedenkstätten der klassischen deutschen Literatur in Weimar sowie aller übrigen öffentlichen und privaten Sammlungen. Bearbeiter der Ausgabe: Dorothea Kuhn, Otfried Wagenbreth, Karl Schneider-Carius. Gesamtredaktion Gerhard Femmel (1967); Bd VIa. Nr 1-302: Zeichnungen aus den Beständen des Goethe- und Schiller-Archivs (1970); Bd VIb. Nr 1-285: Zeichnungen außerhalb der Goethe-Institute der Nationalen Forschungs- und Gedenkstätten der klassischen deutschen Literatur in Weimar. Nachträge. Berichtigungen zu C〈orpus〉 I-VIa. Abschreibungen. Gesamtkonkordanz (1971); Bd VII: Die Zeugnisse (1973).

Zur deutschen Literatur und Geschichte. Ungedruckte Briefe aus Knebels Nachlaß. Hrsg. von Heinrich Düntzer. 2 Bde. Nürnberg 1858.

Susanne Eigenmann: Zwischen ästhetischer Raserei und aufgeklärter Disziplin. Hamburger Theater im späten 18. Jahrhundert. Stuttgart, Weimar 1994. Ereignis Weimar. Anna Amalia, Carl August und das Entstehen der Klassik 1757-1807. Katalog zur Ausstellung im Schlossmuseum Weimar. Hrsg. von der Klassik Stiftung Weimar und dem Sonderforschungsbereich 482 „Ereignis Weimar-Jena. Kultur 
FA/Goethe

FA/Herder

Femmel/Heres

Forster, Werke um 1800" der Friedrich Schiller Universität Jena. Leipzig 2007.

Johann Wolfgang Goethe. Sämtliche Werke. Briefe, Tagebücher und Gespräche. 40 Bde in 2 Abt. 〈Frankfurter Ausgabe〉. Frankfurt a. M. 1985 2011. - I. Abt. Bd 1: Gedichte 1756-1799. Hrsg. von Karl Eibl (1987); Bd 2: Gedichte 1800-1832. Hrsg. von Karl Eibl (1988); Bd 15/2: Italienische Reise. T. 2. Hrsg. von Christoph Michel und HansGeorg Dewitz (1993); Bd 26 und Bd 27: Amtliche Schriften. T. 1 (Bd 26): Geheimes Consilium und andere bis zur Italienreise übernommenen Aufgabengebiete. Hrsg. von Reinhard Kluge (1998). T. 2 (Bd 27): Aufgabengebiete seit der Rückkehr aus Italien. Hrsg. von Irmtraut und Gerhard Schmidt (1999). Bd $26 \mathrm{~K}$ und $7 \mathrm{~K}$ (CD-ROM, 2011): Kommentar zu $B d 26$ und $B d$ 27. Hrsg. von Reinhard Kluge (Bd 26 K), Gerhard und Irmtraut Schmid (Bd $27 \mathrm{~K}$ ); II. Abt. Bd 3: Briefe, Tagebücher und Gespräche vom 3. September 1786 bis 12. Juni 1794. Hrsg. von Karl Eibl (1991).

Johann Gottfried Herder. Werke SFrankfurter Ausgabe). Hrsg. von Martin Bollacher, Jürgen Brummack, Ulrich Gaier u.a. 10 Bde. Frankfurt a. M. 19852000.

Die Gemmen aus Goethes Sammlung. Goethes Sammlungen zur Kunst, Literatur und Naturwissenschaft. Hrsg. von den Nationalen Forschungs- und Gedenkstätten der klassischen deutschen Literatur in Weimar. Bearbeiter der Ausgabe Gerhard Femmel. Katalog Gerald Heres. Leipzig 1977.

Georg Forsters Werke. Sämtliche Schriften, Tagebücher, Briefe. Hrsg. von der Akademie der Wissenschaften zu Berlin (1958-1972), von der Akademie der Wissenschaften der DDR (1973-1990) und von der Berlin-Brandenburgischen Akademie der Wissenschaften (2003). 18 Bde. Berlin 1958-2003. - Bd 9: Ansichten vom Niederrhein, von Brabant, Flandern, Holland, England und Frankreich im April, Mai und Junius 1790. Bearbeitet von Gerhard Steiner (1958); Bd 16: Briefe 1790 bis 1791. Bearbeitet 
Fünfte Nachricht

$G B$ von Brigitte Leuschner und Siegfried Scheibe (1980); Bd 17: Briefe 1792 bis 1794 und Nachträge. Bearbeitet von Klaus-Georg Popp (1989); Bd 18: Briefe an Forster. Bearbeitet von Brigitte Leuschner, Siegfried Scheibe, Horst Fiedler, Klaus-Georg Popp, Annerose Schneider (1982).

Johann Wolfgang von Goethe, Christian Gottlob Voigt: Fünfte Nachricht von dem neuen Bergbaue zu Ilmenau. Wodurch der Erfolg des am Sechsten Junius 1791 eröffneten Gewerkentages bekannt gemacht wird. Weimar 1791.

Johann Wolfgang Goethe: Briefe. Historisch-kritische Ausgabe. Im Auftrag der Klassik-Stiftung Weimar/ Goethe- und Schiller-Archiv / (ab 2017:) In Verbindung mit der Sächsischen Akademie der Wissenschaften zu Leipzig und der Mainzer Akademie der Wissenschaften und der Literatur im Auftrag der Klassik Stiftung Weimar/Goethe- und Schiller-Archiv hrsg. von Georg Kurscheidt, Norbert Oellers und Elke Richter. Berlin 2008ff. - Bd 1 I-II: 23. Mai 1764-30. Dezember 1772. Text und Kommentar. Hrsg. von Elke Richter und Georg Kurscheidt (2008); Bd 2 I-II: Anfang 1773-Ende Oktober 1775. Text und Kommentar. Hrsg. von Georg Kurscheidt und Elke Richter (2008); Bd 3 I-II: 8. November 1775-Ende 1779. Text und Kommentar. Hrsg. von Georg Kurscheidt und Elke Richter unter Mitarbeit von Gerhard Müller und Bettina Zschiedrich (Kommentar) (2014); Bd 6 I-II: Anfang 1785-3. September 1786. Text und Kommentar. Hrsg. von Volker Giel unter Mitarbeit von Susanne Fenske und Yvonne Pietsch (Text); unter Mitarbeit von Yvonne Pietsch, Markus Bernauer und Gerhard Müller (Kommentar) (2010); Bd 7 I-II: 18. September 1786-10. Juni 1788. Text und Kommentar. Hrsg. von Volker Giel unter Mitarbeit von Susanne Fenske und Yvonne Pietsch (Text); unter Mitarbeit von Yvonne Pietsch, Markus Bernauer und Gerhard Müller (Kommentar) (2012); Bd 8 I-II: 20. Juni 1788-Ende 1790. Text und Kommentar. Hrsg. von Volker Giel und Norbert Oellers unter Mitarbeit von 
GB Rep

Gespräche ${ }^{2}$

Gespräche ${ }^{3}$

GJb
Yvonne Pietsch (Text); unter Mitarbeit von Gerhard Müller und Yvonne Pietsch (Kommentar) (2017); Bd 10 I-II: 1794-1795. Text und Kommentar. Hrsg. von Jutta Eckle und Georg Kurscheidt (2019). Johann Wolfgang Goethe. Repertorium sämtlicher Briefe. 1764-1832. Hrsg. von der Klassik Stiftung Weimar/Goethe- und Schiller-Archiv. Bearbeitet von Elke Richter unter Mitarbeit von Andreas Ehlert, Susanne Fenske, Eike Küstner und Katharina Mittendorf. Begründet von Paul Raabe an der Herzog August Bibliothek Wolfenbüttel. Gefördert von der Alfred Krupp von Bohlen und Halbach-Stiftung mit Unterstützung der Deutschen Forschungsgemeinschaft. - Online verfügbar.

Goethes Gespräche. Gesamtausgabe. Neu hrsg. von Flodoard Freiherrn von Biedermann unter Mitwirkung von Max Morris, Hans Gerhard Gräf und Leonhard L. Mackall. 5 Bde. Leipzig 1909-1911. Goethes Gespräche. Eine Sammlung zeitgenössischer Berichte aus seinem Umgang. Aufgrund der Ausgabe und des Nachlasses von Flodoard Freiherrn von Biedermann ergänzt und hrsg. von Wolfgang Herwig. 5 Bde (in 6 Tlen). Zürich und Stuttgart (Bd 3-5: Zürich und München) 1965-1987.

Goethe-Jahrbuch. Bd I-XXXIV. Hrsg. von Ludwig Geiger. Frankfurt a. M. 1880-1913. - Jahrbuch der Goethe-Gesellschaft. Im Auftrage des Vorstandes hrsg. von Hans Gerhard Gräf (Bd 10-21 hrsg. von Max Hecker). Bd 1-9. Leipzig (Bd 8: Weimar) 1914-1922; Bd 10-21. Weimar 1924-1935. Goethe. Bd 1-2: Vierteljahresschrift der GoetheGesellschaft. Neue Folge des Jahrbuchs. Unter Mitwirkung von Ernst Bertram, Rudolf Buttmann, Anton Kippenberg u. a. hrsg. von Hans Wahl. Weimar 1936-1937; Bd 3-9: Viermonatsschrift der Goethe-Gesellschaft. Neue Folge des Jahrbuchs. Unter Mitwirkung von Ernst Bertram, Rudolf Buttmann, Anton Kippenberg u.a. hrsg. von Hans Wahl. Weimar 1938-1944; Bd 10: Neue Folge des Jahrbuchs der Goethe-Gesellschaft. Im Auftrage des Vorstands hrsg. von Hans Wahl. Weimar 1947; Bd 11: Neue 
Folge des Jahrbuchs der Goethe-Gesellschaft. Im Auftrage des Vorstands hrsg. von Hans Wahl † und Andreas B〈runo $\rangle$ Wachsmuth. Weimar 1950; Bd 12-33: Neue Folge des Jahrbuchs der Goethe-Gesellschaft. Im Auftrage des Vorstands hrsg. von Andreas B〈runo $\rangle$ Wachsmuth. Weimar 1951-1971; Goethe Jahrbuch. Bd 89-90: Im Auftrage des Vorstandes der Goethe-Gesellschaft hrsg. von Helmut Holtzhauer. Weimar 1972-1973; Bd 91: Im Auftrage des Vorstandes der Goethe-Gesellschaft hrsg. von Helmut Holtzhauer $f$ und Karl-Heinz Hahn. Weimar 1974; Bd 92-106: Im Auftrage des Vorstandes der Goethe-Gesellschaft hrsg. von Karl-Heinz Hahn. Weimar 1975-1989; Bd 107: Im Auftrage des Vorstandes der Goethe-Gesellschaft hrsg. von Karl-Heinz Hahn † und Jörn Göres. Weimar 1990; Bd 108116: Im Auftrage des Vorstandes (Bd 109ff: Im Auftrag des Vorstands) der Goethe-Gesellschaft hrsg. von Werner Keller. Weimar 1991-1999; Bd 117118: Im Auftrage des Vorstands der Goethe-Gesellschaft hrsg. von Jochen Golz, Bernd Leistner und Edith Zehm. Weimar 2000-2001; Bd 119: Im Auftrag des Vorstands der Goethe-Gesellschaft hrsg. von Jochen Golz und Edith Zehm. Weimar 2002; Bd 120-121: Im Auftrag des Vorstands der GoetheGesellschaft hrsg. von Werner Frick, Jochen Golz und Edith Zehm. Weimar 2003-2004; Bd 122-123: Im Auftrag des Vorstands der Goethe-Gesellschaft hrsg. von Werner Frick, Jochen Golz und Edith Zehm. Göttingen 2005-2006; Bd 124-127: Im Auftrag des Vorstands der Goethe-Gesellschaft hrsg. von Werner Frick, Jochen Golz, Albert Meier und Edith Zehm. Göttingen 2007-2010; Bd 128131: Im Auftrag der Goethe-Gesellschaft hrsg. von Jochen Golz, Albert Meier und Edith Zehm. Göttingen 2011-2014; Bd 132: Im Auftrag der GoetheGesellschaft hrsg. von Jochen Golz und Edith Zehm. Göttingen 2015; Bd 133-135: Im Auftrag des Vorstands der Goethe-Gesellschaft hrsg. von Frieder von Ammon, Jochen Golz und Edith Zehm. Göttingen 2016-2018. 
Goethe-Christiane

Goethe-Friedrich von Stein

Goethe in Trier und Luxemburg

Goethe-Jacobi ${ }^{1}$

Goethe-Knebel

Goethe-Lavater ${ }^{3}$

Goethe-Meyer

Goethes Briefe an Leipziger Freunde Goethe und Bergbau

Goethe und Ilmenau

Goethe und Kreis von Münster
Goethes Briefwechsel mit seiner Frau. Hrsg. von Hans Gerhard Gräf. 2 Bde (Erster Band 17921806. Zweiter Band 1807-1816). Frankfurt a. M. 1916.

Briefe Goethes und dessen Mutter an Friedrich Freiherr von Stein, nebst einigen Beilagen. Hrsg. von Johann Jacob Heinrich Ebers und August Kahlert. Leipzig 1846.

Goethe in Trier und Luxemburg. 200 Jahre Campagne in Frankreich 1792. Katalog der Ausstellung der Stadtbibliothek Trier, der Nationalbibliothek Luxemburg und der Stiftung Weimarer Klassik. Trier, Luxemburg 1992.

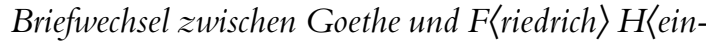
rich〉 Jacobi. Hrsg. von Max Jacobi. Leipzig 1846. Briefwechsel zwischen Goethe und Knebel. (17741832.) 〈Hrsg. von G[ottschalk] E[duard] Guhrauer.〉 2 Tle. Leipzig 1851.

Goethe und Lavater. Briefe und Tagebücher. Hrsg. von Heinrich Funck (SchrGG 16). Weimar 1901. Goethes Briefwechsel mit Heinrich Meyer. Hrsg. von Max Hecker. 4 Bde. Weimar 1917-1932. - Bd 1: Juli 1788 bis Juni 1797 (1917; SchrGG 32). Bd 2: Juni 1797 bis Dezember 1820 (1919; SchrGG 34). Bd 3: Januar 1821 bis März 1832 (1922; SchrGG 35.1). Bd 4: Register zu Bd 1 - 3 (1932; SchrGG 35.2).

Goethes Briefe an Leipziger Freunde. Hrsg. von Otto Jahn. Leipzig 1849.

Otfried Wagenbreth: Goethe und der Ilmenauer Bergbau. Weimar 1983.

Goethe und Ilmenau. Unter Benutzung zahlreichen unveröffentlichten Materials dargestellt von Julius Voigt. Mit einem Geleitwort von Karl-Heinz Hahn und einem Nachwort von Rosalinde Gothe. Reprint der Originalausgabe Leipzig 1912. Leipzig 1990. Goethe und der Kreis von Münster. Zeitgenössische Briefe und Aufzeichnungen. In Zusammenarbeit mit Waltraud Loos hrsg. von Erich Trunz (Veröffentlichungen der Historischen Kommission West- 
falens XIX. Westfälische Briefwechsel und Denkwürdigkeiten. Bd 6). Münster 1971.

Goethe und Soemmerring Goethe und Soemmerring. Briefwechsel 17841828. Textkritische und kommentierte Ausgabe. Bearbeitet und hrsg. von Manfred Wenzel (Soemmerring-Forschungen. Beiträge zur Naturwissenschaft und Medizin der Neuzeit. Hrsg. von Günter Mann, Jost Benedum, Werner F. Kümmel. Band V). Stuttgart, New York 1988.

Goethe und Werther ${ }^{1} \quad$ Goethe und Werther. Briefe Goethe's, meistens aus seiner Jugendzeit, mit erläuternden Documenten. Hrsg. von A〈ugust〉 Kestner. Stuttgart und Tübingen 1854.

Goethe-Voigt ${ }^{1}$

Goethe-Voigt ${ }^{2}$

Goschen

Goethes Briefe an Christian Gottlob Voigt. Mit Voigts Bildniß. Hrsg. von Otto Jahn. Leipzig 1868. Goethes Briefwechsel mit Christian Gottlob Voigt. 4 Bde. Bearbeitet und hrsg. von Hans Tümmler (ab Bd 3 unter Mitwirkung von Wolfgang Huschke; SchrGG 53-56). Weimar 1949 (Bd 1), 1951 (Bd 2), 1955 (Bd 3), 1962 (Bd 4).

Das Leben Georg Joachim Göschens. Von seinem Enkel Viscount Goschen. Deutsche, vom Verfasser bearbeitete Ausgabe, übersetzt von Th〈omas $\rangle$ A. Fischer. 2 Bde. Leipzig 1905.

Grimm

Deutsches Wörterbuch von Jacob und Wilhelm Grimm. 16 Bde. Leipzig 1854-1961.

GT Johann Wolfgang Goethe: Tagebücher. Historisch-kritische Ausgabe. Im Auftrag der Stiftung Weimarer Klassik 〈ab Bd V (2007): Klassik Stiftung Weimar〉 hrsg. von Jochen Golz unter Mitarbeit von Wolfgang Albrecht, Andreas Döhler und Edith Zehm. Bd Iff. Stuttgart, Weimar 1998ff. - Bd I 1-2: 1775-1787. Text und Kommentar. Hrsg. von Wolfgang Albrecht und Andreas Döhler (1998); Bd II 1: 1790-1800. Text. Hrsg. von Edith Zehm (2000); Bd II 2: 1790-1800. Kommentar. Hrsg. von Wolfgang Albrecht und Edith Zehm (2000); Bd III 1-2: 1801-1808. Text und Kommentar. Hrsg. von Andreas Döhler (2004); Bd IV 1-2: 1809-1812. Text und Kommentar. Hrsg. von Edith Zehm, Sebastian Mangold und Ariane Ludwig (2008); Bd V 
$G W b$

Hagen

Harnack, Nachgeschichte

Hartung,

Reichardts Entlassung

Haym

$H B$
1-2: 1813-1816. Text und Kommentar. Hrsg. von Wolfgang Albrecht (2007); Bd VI 1-2: 18171818. Text und Kommentar. Hrsg. von Andreas Döhler (2014); Bd VII 1-2: 1819-1820. Text und Kommentar. Hrsg. von von Edith Zehm, Sebastian Mangold und Ariane Ludwig (2014); Bd VIII 1-2: 1821-1822. Text und Kommentar. Hrsg. von Wolfgang Albrecht (2015).

Goethe Wörterbuch. Bd 1-2. Hrsg. von der Akademie der Wissenschaften der DDR, der Akademie der Wissenschaften in Göttingen und der Heidelberger Akademie der Wissenschaften. Stuttgart, Berlin, Köln, Mainz 1978-1989. - Bd 3ff. Hrsg. von der Berlin-Brandenburgischen Akademie der Wissenschaften, der Akademie der Wissenschaften in Göttingen und der Heidelberger Akademie der Wissenschaften. Stuttgart, Berlin, Köln $1998 f f$.

Die Drucke von Goethes Werken. Hrsg. von der Akademie der Wissenschaften der DDR. Bearbeitet von Waltraud Hagen. 2., durchgesehene Aufl. Berlin 1983.

Zur Nachgeschichte der italienischen Reise. Goethes Briefwechsel mit Freunden und Kunstgenossen in Italien 1788-1790. Hrsg. von Otto Harnack (SchrGG 5). Weimar 1890.

Günter Hartung: Reichardts Entlassung. In: Wissenschaftliche Zeitschrift der Martin-Luther-Universität Halle-Wittenberg. Gesellschafts- und Sprachwissenschaftliche Reihe. Jg X, H. 4 [Mai]. Halle 1961, S. 971-980.

Rudolf Haym: Herder. 2 Bde. Berlin 1954.

Johann Gottfried Herder: Briefe. Gesamtausgabe 1763-1803. Unter Leitung von Karl-Heinz Hahn hrsg. von den Nationalen Forschungs- und Gedenkstätten der klassischen deutschen Literatur in Weimar $\langle a b$ Bd 10: Hrsg. von der Klassik Stiftung Weimar〉 (Goethe- und Schiller-Archiv). 17 Bde $\langle B d 1-8$ : Bearbeitet von Wilhelm Dobbek † und Günter Arnold. Bd 9-17: Bearbeitet von Günter Arnold $\rangle$. Weimar 1977-2014. - Bd 6: August 1788-Dezember 1792 (1989); Bd 7: Januar 1793-Dezember 1798 
(1982); Bd 9: Nachträge und Ergänzungen 17631803 (1988); Bd 13: Kommentar zu Band 6 (2009); Bd 14: Kommentar zu Band 7 (2009).

Henninger, Bethmann Wolfgang Henninger: Johann Jakob von Bethmann. 1717-1792. Kaufmann, Reeder und kaiserlicher Konsul in Bordeaux. 2 Tle (Dortmunder Historische Studien. Bd 4. Tle 1 und 2) Bochum 1993.

Herting, Maximilian Jacobi Carl Wigand Maximilian Jacobi ein deutscher Arzt (1775-1858). Ein Lebensbild nach Briefen und anderen Quellen von Sanitätsrat Dr. Johannes Herting. Görlitz 1930.

Heyderhoff,

Freundschaftsbriefe

Hirzel,

Goethe-Bibliothek 1874

Holtei, Goethe in

Breslau 1

Holtei, Goethe in

Breslau 2

IR I; II; III

Italiänische Reise I, II, III
Die Hausgeister von Pempelfort. Familien- und Freundschaftsbriefe des Jacobi-Hauses gesammelt und herausgegeben von Julius Heyderhoff. Düsseldorf 1939.

Salomon Hirzel: Neuestes Verzeichniß einer GoetheBibliothek (1767-1874). Leipzig 1874.

Goethe in Breslau. Auszüge aus des Freiherrn von Schuckmann Briefen an Capellmeister Fr〈iedrich〉 Reichardt. Mitgetheilt von Karl Holtei. In: Westermann's Illustrirte Deutsche Monatshefte. Nro. 1 der zweiten Folge. Der ganzen Reihe Nro. 97. October 1864. Braunschweig 1864, S. 76-84 (Westermann's Jahrbuch der Illustrirten Deutschen Monatshefte. Ein Familienbuch für das gesammte geistige Leben der Gegenwart. Siebzehnter Band. Der neuen Folge erster Band. October 1864-März 1865).

Goethe in Breslau. Mitgetheilt von Karl Holtei. II. (Vier Briefe von Goethe an Freih〈errn $\rangle\langle\langle o n\rangle$ Schuckmann.) In: Westermann's Illustrirte Deutsche Monatshefte. Nro. 8 der zweiten Folge. Der ganzen Reihe Nro. 104. Mai 1865. Braunschweig 1865, S. 154-157 (Westermann's Jahrbuch der Illustrirten Deutschen Monatshefte. Ein Familienbuch für das gesammte geistige Leben der Gegenwart. Achtzehnter Band. Der neuen Folge zweiter Band. April-September 1865).

Italiänische Reise. I. II. III. (WA I 30-32.)

Aus meinem Leben. Von Goethe. Zweyter Abtheilung Erster Theil. Auch ich in Arcadien! Stuttgard, Tübingen 1816. - Aus meinem Leben. Von Goethe. Zwey- 
ter Abtheilung Zweyter Theil. Auch ich in Arcadien! Stuttgard, Tübingen 1817. - Zweiter Römischer Aufenthalt vom Juni 1787 bis April 1788. Goethe's Werke. Vollständige Ausgabe letzter Hand. Neunundzwanzigster Band. Stuttgart, Tübingen 1829.

Friedrich Heinrich Jacobi: Briefwechsel. Gesamtausgabe. Hrsg. von Michael Brüggen und Siegfried Sudhof (ab Bd 3: Begründet von Michael Brüggen und Siegfried Sudhof t. Hrsg. von Michael Brüggen, Heinz Gockel und Peter-Paul Schneider; ab Bd 4: Gesamtausgabe der Bayerischen Akademie der Wissenschaften. Begründet von Michael Brüggen und Siegfried Sudhof †. Hrsg. von Michael Brüggen und Heinz Gockel; ab Bd 5: Hrsg. von Walter Jaeschke).

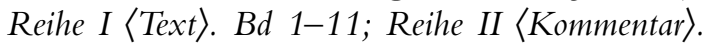
$B d$ 1-5 und 9. Stuttgart-Bad Cannstatt 19812017-Bd I 1: Briefwechsel 1762-1775. Hrsg. von Michael Brüggen und Siegfried Sudhoff in Zusammenarbeit mit Peter Bachmeier, Reinhard Lauth und Peter-Paul Schneider (1981); Bd II 1: Briefwechsel 1762-1775. Kommentar von Michael Brüggen und Reinhard Lauth unter Mitwirkung von Peter Bachmeier, Albert Mues und Isabel Schmidt (1989); Bd I 2: Briefwechsel 1775-1781. Hrsg. von Peter Bachmeier, Michael Brüggen, Reinhard Lauth und Siegfried Sudhoff + in Zusammenarbeit mit Peter-Paul Schneider (1983); Bd II 2: Briefwechsel 17751781. Kommentar von Michael Brüggen unter Mitwirkung von Reinhard Lauth sowie Albert Mues und Gudrun Schury (1997); Bd I 3: Briefwechsel 1782-1784. Hrsg. von Peter Bachmeier, Michael Brüggen, Heinz Gockel, Reinhard Lauth und PeterPaul Schneider (1987); Bd II 3: Briefwechsel 1782-1784. Kommentar von Michael Brüggen unter Mitwirkung von Albert Mues und Gudrun Schury (2001); Bd I 4: Briefwechsel 1785. Nachtrag zum Briefwechsel 1764-1784. Hrsg. von Albert Mues, Gudrun Schury und Jutta Torbi (2003); Bd II 4.1: Briefwechsel 1785. Nachtrag zum Briefwechsel 1764-1784. Kommentar begonnen von Michael Brüggen unter Mitwirkung von Albert Mues, Gudrun 
Schury und Jutta Torbi † weitergeführt von Peter Kriegel und Rebecca Paimann. Teilbd 1: Nr 1108-1230 (2013); Bd II 4.2: Briefwechsel 1785. Nachtrag zum Briefwechsel 1764-1784. Kommentar begonnen von Michael Brüggen unter Mitwirkung von Albert Mues, Gudrun Schury und Jutta Torbi † weitergeführt von Peter Kriegel und Rebecca Paimann. Teilbd 2: Nr 1231-1306 und 23.01 und 1106.1 (2013); Bd I 5: Briefwechsel 1786. Hrsg. von Walter Jaeschke und Rebecca Paimann. Unter Mitarbeit von Albert Mues, Gudrun Schury und Jutta Torbi (2005); Bd II 5,1-2. Briefwechsel 1786. Kommentar von Irmgard Huthmacher (2014); Bd I 6: Briefwechsel Januar bis November 1787. Hrsg. von Jürgen Weyenschops. Unter Mitarbeit von Albert Mues, Gudrun Schury und Jutta Torbi † (2012); Bd I 7: Briefwechsel November 1787 bis Juni 1788. Hrsg. von Jürgen Weyenschops, Albert Mues, Gudrun Schury Jutta Torbi † und Walter Jaeschke (2012); Bd I 8: Briefwechsel Juli 1788 bis Dezember 1790. Hrsg. von Manuela Köppe (2015); Bd I 9: Briefwechsel Januar 1791 bis Mai 1792. Hrsg. von Walter Jaeschke und Rebecca Paimann (2015); Bd II 9: Briefwechsel Januar 1791 bis Mai 1792. Kommentar von Walter Jaeschke unter Mitwirkung von Rebecca Paimann und Konstanze Sommer (2016); Bd I 10: Briefwechsel Juni 1792 bis September 1794. Nachtrag zum Briefwechsel 1769-1789. Hrsg. von Walter Jaeschke und Rebecca Paimann (2015); Bd I 11: Briefwechsel Oktober 1794 bis Dezember 1798. Hrsg. von Walter Jaeschke und Birgit Sandkaulen (2017).

$J b F D H$

Jahrbuch des Freien Deutschen Hochstifts. 〈Hrsg. von Otto Heuer.) Frankfurt 1902-1925. - Im Auftrag der Verwaltung hrsg. von Ernst Beutler. Frankfurt 1926-1930; Halle 1931-1940. - Hrsg. von Detlef Lüders. Tübingen 1962-1982. - Hrsg. von Arthur Henkel. Tübingen 1983. - Hrsg. von Christoph Perels. Tübingen 1984-2002. Hrsg. von Anne Bohnenkamp und Christoph Perels. Tübingen 2003. Hrsg. von Anne Bohnenkamp. Tübingen $2004 f f$. 
Journal von Tiefurt

Kant $A A X I, 2$

Katalog Schloss

Knebel, Nachlaß und Briefwechsel

Krünitz, Enzyklopädie

Kruse, Lips
Das Journal von Tiefurt. Mit einer Einleitung von Bernhard Suphan hrsg. von Eduard von der Hellen (SchrGG 7). Weimar 1892.

Kant's gesammelte Schriften. Hrsg. von der Königlich Preußischen Akademie der Wissenschaften. Bd XI. 2. Abteilung: Briefwechsel. Bd 2: 1789-1794. Berlin 1900.

Katalog der Zeichnungen, Graphiken und Gemälde zur Bangeschichte des Weimarer Schlosses. In: Rolf Bothe: Dichter, Fürst und Architekten. Das Weimarer Residenzschloß vom Mittelalter bis zum Anfang des 19. Jahrhunderts. Ostfildern-Ruit 2000, S. 114-145.

Keudell, Goethe-Bibliothek Goethe als Benutzer der Weimarer Bibliothek. Ein Verzeichnis der von ihm entliehenen Werke. Bearbeitet von Elise von Keudell. Hrsg. mit einem Vorwort von Prof. Dr. Werner Deetjen. Weimar 1931.

$K\langle a r l\rangle L\langle u d w i g\rangle$ von Knebel's literarischer Nachlaß

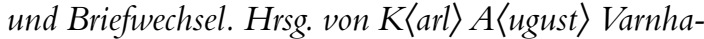
gen von Ense und Th〈eodor $\rangle$ Mundt. 3 Bde. Leipzig 1835-1836.

Oekonomische Encyklopädie, oder allgemeines System der Land-Haus- und Staats-Wirthschaft, in alphabetischer Ordnung; von D. Johann Georg Krünitz. 242 Tle. Berlin 1773-1858. - T. 81-123: D. Johann Georg Krünitz's oekonomisch-technologische Encyclopädie, oder allgemeines System der Staats-, Stadt-, Haus- u. Landwirthschaft, und der Kunstgeschichte; Zuerst fortgesetzt von Friedrich Jakob Floerken, nunmehr von Heinrich Gustav Floerke. Berlin 1801-1813. - T. 197-242: Oekonomische Encyklopädie, oder allgemeines System der Land-Haus- und Staats-Wirthschaft, in alphabetischer Ordnung; Aus dem Französ〈ischen〉 übersetzt und mit Anmerkungen und Zusätzen vermehrt von Georg Krünitz. Früher fortgesetzt von Friedrich Jakob und Heinrich Gustav Floerke, und jetzt von Johann Wilhelm David Korth, und Ludwig Koßarski. Berlin 1849-1858.

Joachim Kruse: Johann Heinrich Lips. 1758-1817. Ein Zürcher Kupferstecher zwischen Lavater und 
Goethe. [Ausstellung:] 30.7.-5.11.1989. Kunstsammlungen der Veste Coburg. Coburger Landesstiftung (Kataloge der Kunstsammlungen der Veste Coburg). Coburg 1989.

Goethe: Die Schriften zur Naturwissenschaft. Vollständige mit Erläuterungen versehene Ausgabe. Im Auftrage der Deutschen Akademie der Naturforscher (Leopoldina) zu Halle begründet von Karl Lothar Wolf und Wilhelm Troll. Hrsg. von Dorothea Kuhn, Wolf von Engelhardt, 〈seit 2005〉 Irmgard Müller und 〈seit 2012〉 Friedrich Steinle. Weimar 1947ff. I. Abteilung: Texte. 11 Bde. 1947-1970. II. Abteilung: Ergänzungen und Erläuterungen. 10 Bde (in 18 Tlen). 1959-2011. III. Abteilung: Verzeichnisse und Register. 2014-2019. - Bd I 1: Schriften zur Geologie und Mineralogie 1770-1810. Hrsg. von Günther Schmid (1947); Bd I 2: Schriften zur Geologie und Mineralogie 1812-1832. Hrsg. von Günther Schmid (1949); Bd I 3: Beiträge zur Optik und Anfänge der Farbenlehre 1790-1808. Hrsg. von Rupprecht Matthaei (1951); Bd I 4: Zur Farbenlehre. Widmung, Vorwort und Didaktischer Teil. Bearbeitet von Rupprecht Matthaei (1955); Bd I 5: Zur Farbenlehre. Polemischer Teil. Bearbeitet von Rupprecht Matthaei (1958); Bd I 6: Zur Farbenlehre. Historischer Teil. Bearbeitet von Dorothea Kuhn (1957); Bd I 7: Zur Farbenlehre. Anzeige und Übersicht, statt des supplementaren Teils und Erklärung der Tafeln. Bearbeitet von Rupprecht Matthaei (1957); Bd I 8: Naturwissenschaftliche Hefte. Bearbeitet von Dorothea Kuhn (1962); Bd I 9: Morphologische Hefte. Bearbeitet von Dorothea Kuhn (1954); Bd I 10: Aufsätze, Fragmente, Studien zur Morphologie. Bearbeitet von Dorothea Kuhn (1964); Bd I 11: Aufsätze, Fragmente, Studien zur Naturwissenschaft im Allgemeinen. Bearbeitet von Dorothea Kuhn und Wolf von Engelhardt (1970); Bd II $1 A$ und II 1B: Zur Naturwissenschaft im Allgemeinen. Bearbeitet von Jutta Eckle (2011); Bd II 2: Zur Meteorologie und Astronomie. Bearbeitet von Gisela Nickel (2005); Bd II 3: Beiträge zur Optik und An- 
fänge der Farbenlehre. Bearbeitet von Rupprecht Matthaei und Dorothea Kuhn (1961); Bd II 4: Zur Farbenlehre. Didaktischer Teil und Tafeln. Bearbeitet von Rupprecht Matthaei und Dorothea Kuhn (1973); Bd II 5A: Zur Farbenlehre. Polemischer Teil. Bearbeitet von Horst Zehe (1992); Bd II 5B/1 und II 5B/2: Zur Farbenlehre und Optik nach 1810 und zur Tonlehre. Bearbeitet von Thomas Nickol unter Mitwirkung von Dorothea Kuhn und Horst Zehe (2007); Bd II 6: Zur Farbenlehre. Historischer Teil. Bearbeitet von Dorothea Kuhn und Karl Lothar Wolf (1959); Bd II 7: Zur Geologie und Mineralogie. Von den Anfängen bis 1805. Bearbeitet von Wolf von Engelhardt unter Mitwirkung von Dorothea Kuhn (1989); Bd II 8A: Zur Geologie und Mineralogie. Von 1806 bis 1820. Bearbeitet von Wolf von Engelhardt unter Mitwirkung von Dorothea Kuhn (1997); Bd II 8B/1 und II 8B/2: Zur Geologie und Mineralogie. Von 1821 bis 1832. Bearbeitet von Wolf von Engelhardt unter Mitwirkung von Dorothea Kuhn (1999); Bd II 9A: Zur Morphologie. Von den Anfängen bis 1795. Bearbeitet von Dorothea Kuhn (1977); Bd II 9B: Zur Morphologie. Von 1796 bis 1815. Bearbeitet von Dorothea Kuhn (1986); Bd II 10A: Zur Morphologie. Von 1816 bis 1824. Bearbeitet von Dorothea Kuhn (1995); Bd II 10B/1 und II 10B/2: Zur Morphologie. Von 1825 bis 1832. Bearbeitet von Dorothea Kuhn (2004); Bd III 1: Verzeichnisse. Bearbeitet von Bastian Röther und Uta Monecke (2014); Bd III 2: Register. Bearbeitet von Carmen Götz, Simon Rebohm und Bastian Röther (2019).

Lachmann/Muncker Gotthold Ephraim Lessings sämtliche Schriften. Hrsg. von Karl Lachmann. Dritte, auf's neue durchgesehene und vermehrte Ausgabe, besorgt durch Franz Muncker. 23 Bde. (Bd 1-11 Stuttgart; Bd 12-21 Leipzig; Bd 22-23 Berlin und Leipzig.) 1886-1924.

Lichtenberg, Briefwechsel Georg Christoph Lichtenberg: Briefwechsel. Im Auftrag der Akademie der Wissenschaften zu Göttingen hrsg. von Ulrich Joost und Albrecht Schöne. 5 Bde (in 6 Tlen). München 1983-2004. - Bd 2: 
Luther-Bibel 1772 AT/Apokryphen/NT

Lüttwitz,

Biographie Schuckmann

MA/Goethe
1780-1784. Hrsg. von Ulrich Joost und Albrecht Schöne (1985); Bd 3: 1785-1792. Hrsg. von Ulrich Joost und Albrecht Schöne (1990); Bd 4: 1793-1799 und Undatiertes. Hrsg. von Ulrich Joost und Albrecht Schöne unter Mitwirkung von Julia Hoffmann (1992).

Biblia, / Das ist: / Die ganze / Heilige Schrift / Alten und Neuen / Testamentes, / Nach der deutschen Uebersetzung / D. Martin Luthers, / mit vorgesetztem kurzen / Inhalt eines jeden Capitels, / wie auch mit richtigen / Summarien und vielen Schrift-Stellen / auf das allersorgfältigste versehen, nach den bewährtesten und neuesten Editionen / mit grossem Fleisse ausgefertiget. I Samt / einer Vorrede /von / Hieronymo Burckhardt, I der Heil. Schrift Doctor. I Basel 1772. (Vgl. Ruppert, 384, Nr 2604.)

〈Hans Ernst〉 von Lüttwitz: Biographie des kö-

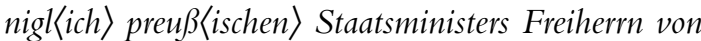
Schuckmann. Leipzig 1835.

Johann Wolfgang Goethe. Sämtliche Werke nach Epochen seines Schaffens 〈Münchner Ausgabe〉. Hrsg. von Karl Richter in Zusammenarbeit mit Herbert G. Göpfert, Norbert Miller und Gerhard Sauder (Bd 7, 11 I 1, 11 I 2, 11 II, 13 I, 13 II, 15, 17, 18 I, 18 II, 20 I, 20 II, 20 III. Hrsg. von Karl Richter in Zusammenarbeit mit Herbert G〈eorg〉 Göpfert, Norbert Miller, Gerhard Sauder und Edith Zehm). 21 Bde. München, Wien 1985-1998. - Bd 3 II: Italien und Weimar 1786-1790. Hrsg. von Hans J. Becker, Hans-Georg Dewitz, Norbert Miller, Gerhard H. Müller, John Neubauer, Hartmut Reinhardt und Irmtraut Schmidt (1990); Bd 4 I: Wirkungen der Französischen Revolution 1791-1797. 〈T. $\rangle$ 1. Hrsg. von Reiner Wild (1988); Bd 4 II: Wirkungen der Französischen Revolution 1791-1797. 〈T.〉2. Hrsg. von Klaus H. Kiefer, Hans J. Becker, Gerhard H. Müller, John Neubauer und Peter Schmidt (1986); Bd 13 I: Die Jahre 1820-1826. Hrsg. von Gisela Henckmann und Irmela Schneider (1992); Bd 14: Autobiographische Schriften der frühen Zwanzigerjahre. 
Meier,

Unterhaltungsliteratur

Meyer, Schröder Leben

Mommsen

Müller, Universität Jena

Muller, Goethe in Mertert

$N A$
Hrsg. von Reiner Wild (1986); Bd 15: Italienische Reise. Hrsg. von Andreas Beyer und Norbert Miller (1992); Bd 18 II: Letzte Jahre 1827-1832. 〈T.〉 2. Hrsg. von Johannes John, Hans J. Becker, Gerhard H. Müller, John Neubauer und Irmtraut Schmid (1996).

Andreas Meier: „Die triviale Klassik' - Unterhaltungsliteratur als kulturelles Komplement. In: Christian August Vulpius: Eine Korrespondenz zur Kulturgeschichte der Goethezeit. Hrsg. von Andreas Meier. Bd 1: Brieftexte. Berlin, New York 2003, S. XI-CLXXXVII.

Friedrich Ludwig Schröder: Beitrag zur Kunde des Menschen und des Künstlers von F. L. W. Meyer. Friedrich Ludwig Schröders Leben. 2 Tle (T. 1; T. 2. Abt. 1; T. 2. Abt. 2). Hamburg 1819.

Momme Mommsen unter Mitwirkung von Katharina Mommsen: Die Entstehung von Goethes Werken in Dokumenten. Bd 1: Abaldemus bis Byron. Bd 2: Cäcilia bis Dichtung und Wahrheit. Berlin 1958. Gerhard Müller: Vom Regieren zum Gestalten. Goethe und die Universität Jena (Ereignis Weimar-Jena. Kultur um 1800. Ästhetische Forschungen. Hrsg. von Klaus Manger. Bd 6). Heidelberg 2006.

Jean-Claude Muller: Goethe in Mertert (21.-22. Oktober 1792). Ein neuentdeckter Brief. In: Les cahiers luxembourgeois. Zu J. W. Goethe. Aus Luxemburg. Hrsg. von Nic Weber. Mai 1999. Luxemburg 1999.

Schillers Werke. Nationalausgabe. Bd 1: Im Auftrag des Goethe- und Schiller-Archivs, des Schiller-Nationalmuseums und der Deutschen Akademie hrsg. von Julius Petersen $†$ und Gerhard Fricke. Weimar 1943. - Bd 3, 5, 8, 9, 13, 16, 22, 23, 27: Im Auftrag des Goethe- und Schiller-Archivs und des Schiller-Nationalmuseums hrsg. von Julius Petersen $\dagger$ und Hermann Schneider. Weimar 1948-1958. - Bd 6, $7 \mathrm{I}, 11,17,18,20,25,28,29,30,35,36 I$, 36 II, 38 I, 42: Begründet von Julius Petersen. Hrsg. im Auftrag der Nationalen Forschungs- und Gedenkstätten der klassischen deutschen Literatur in Weimar 
(Goethe- und Schiller-Archiv) und des Schiller-Nationalmuseums in Marbach von Lieselotte Blumenthal und Benno von Wiese. Weimar 1961-1979. - Bd 2 I, 2 II A, 4, 7 II, 10, 12, 24, 31, 32, 33 I, 34 I, 37 I, 37 II, 39 I, 40 I: Begründet von Julius Petersen. Fortgeführt von Lieselotte Blumenthal und Benno von Wiese. Hrsg. im Auftrag der Nationalen Forschungs- und Gedenkstätten der klassischen deutschen Literatur in Weimar (Goethe- und Schiller-Archiv) und des Schiller-Nationalmuseums in Marbach von Norbert Oellers und Siegfried Seidel. Weimar 1980-1991. - Bd 15 I, 26: 1940 begründet von Julius Petersen. Fortgeführt von Lieselotte Blumenthal und Benno von Wiese. Hrsg. im Auftrag der Stiftung Weimarer Klassik und des Schiller-Nationalmuseums Marbach von Norbert Oellers und Siegfried Seidel $t$. Weimar 1992-1993. - Bd 2 II B, 5 N, 8 N 1, 8 N 2, $8 N 3,9 N 1,9 N 2,15 \mathrm{II}, 19 \mathrm{I}, 33 \mathrm{II}, 34 \mathrm{II}$, 40 II, 41 I, 41 II A: 1940 begründet von Julius Petersen. Fortgeführt von Lieselotte Blumenthal, Benno von Wiese, Siegfried Seidel. Hrsg. im Auftrag der Stiftung Weimarer Klassik 〈Bd 41 II A (2006): Klassik Stiftung Weimar $\rangle$ und des Schiller-Nationalmuseums in Marbach von Norbert Oellers. Weimar $1993 \mathrm{ff}$. $B d$ 1: Gedichte in der Reihenfolge ihres Entstehens 1776-1799. Hrsg. von Julius Petersen † und Friedrich Beißner (1943); Bd 15 I: Übersetzungen aus dem Griechischen und Lateinischen. Hrsg. von Heinz Gerd Ingenkamp (1993); Bd 26: Briefwechsel. Schillers Briefe 1.3.1790-17.5.1794. Hrsg. von Edith Nahler und Horst Nahler (1992); Bd 34 I: Briefwechsel. Briefe an Schiller 1.3.1790-24.5.1794. Hrsg. von Ursula Naumann (1991); Bd 34 II: Briefwechsel. Briefe an Schiller 1.3.1790-24.5.1794. Hrsg. von Ursula Naumann (1997); Bd 36 I: Briefwechsel. Briefe an Schiller 1.11.1795-31.3.1797. Hrsg. von Norbert Oellers (1972).

Neuper, Vorlesungsangebot Das Vorlesungsangebot an der Universität Jena von Jena 1749 bis 1854. Hrsg. von Horst Neuper unter Mitarbeit von Katarina Kühn und Matthias Müller. 2 Bde. Weimar 2003. 
Neuß, Giebichensteiner

Dichterparadies

Pasqué

Pfeiffer-Belli

Polianski, Ästhetisierung Pflanzenkunde

Politischer Briefwechsel

Post-Bericht 1792

Prescher,

Goethes Sammlungen

Priestley
Erich Neuß: Das Giebichensteiner Dichterparadies. Johann Friedrich Reichardt und die Herberge der Romantik. 3. Aufl. Hrsg. vom Landesheimatbund Sachsen-Anhalt e. V. Halle (Saale) 2007.

Goethe's Theaterleitung in Weimar. In Episoden und Urkunden dargestellt von Ernst Pasqué. 2 Bde. Leipzig 1863.

Johann Caspar Goethe / Cornelia Goethe / Catharina Elisabeth Goethe: Briefe aus dem Elternhaus. Erster Ergänzungsband der Goethe-Gedenkausgabe. Hrsg. von Wolfgang Pfeiffer-Belli. Zürich, Stuttgart 1960.

Igor J. Polianski: Die Kunst, die Natur vorzustellen. Die Ästhetisierung der Pflanzenkunde um 1800 und Goethes Gründung des botanischen Gartens zu Jena im Spannungsfeld kunstheoretischer und botanischer Diskussionen der Zeit (Minerva. Jenaer Schriften zur Kunstgeschichte. Bd 14. Hrsg. von Franz-Joachim Verspohl \& Ulrich Müller in Zusammenarbeit mit Karl-Michael Platen). Köln 2004.

Politischer Briefwechsel des Herzogs und Großherzogs Carl August von Weimar. Hrsg. von Willy Andreas. Bearbeitet von Hans Tümmler. 3 Bde (Quellen zur deutschen Geschichte des 19. und 20. Jahrhunderts. Bd 37-39). Stuttgart 1954 und 1958. Göttingen 1973. - Bd 1: Von den Anfängen der Regierung bis zum Ende des Fürstenbundes 1778-1790 (1954); Bd 2: Vom Beginn der Revolutionskriege bis in die Rheinbundszeit 1791-1807 (1958); Bd 3: Von der Rheinbundzeit bis zum Ende der Regierung 1808-1828 (1973).

Post-Bericht, wie die Posten allhier abgehen und ankommen. In: Neuverbesserter Calender, für alle Stände, auf das Schalt-Jahr 1792. Weimar [1791], S. $\langle 36\rangle$.

Hans Prescher: Goethes Sammlungen zur Mineralogie, Geologie und Paläontologie. Katalog. Berlin 1978.

Dr. Joseph Priestleys Mitgliedes der Königl. Großbrittanischen Gesellschaft der Wissenschaften Geschichte und gegenwärtiger Zustand der Optik, vorzüglich in 
Pröpper,

Bühnenwerke Reichardts

$\mathrm{QuZ}$

$R A$
Absicht auf den physikalischen Theil dieser Wissenschaft. Aus dem Englischen übersetzet und mit Anmerkungen und Zusätzen begleitet von Georg Simon Klügel Professor der Mathematik zu Helmstädt, Correspondenten der Königl. Gesellschaft der Wissenschaften zu Göttingen, und Mitgliede einiger anderer gelehrten Gesellschaften. Zween Theile. Leipzig 1776.

Rolf Pröpper: Die Bühnenwerke Johann Friedrich Reichardts (1752-1814). Ein Beitrag zur Geschichte der Oper in der Zeit des Stilwandels zwischen Klassik und Romantik. In Verbindung mit dem Verzeichnis der literarischen Werke und einem Katalog der Bühnenwerke Johann Friedrich Reichardts. - Bd 1: Textteil; Bd 2: Werkverzeichnis (Abhandlungen zur Kunst-, Musik- und Literaturwissenschaft. Bd 25). Bonn 1965.

Quellen und Zeugnisse zur Druckgeschichte von Goethes Werken. Hrsg. von der Deutschen Akademie der Wissenschaften zu Berlin. (T. 2-4: Hrsg. vom Zentralinstitut für Literaturgeschichte der Akademie der Wissenschaften der DDR). 4 Tle. Berlin 1966-1984. - T. 1: Gesamtausgaben bis 1822. Bearbeiter des Bandes: Waltraud Hagen unter Mitarbeit von Edith Nahler (1966); T. 2: Die Ausgabe letzter Hand. Bearbeiter des Bandes: Waltraud Hagen (1982); T. 3: Die nachgelassenen Werke und die Quartausgabe. Bearbeiter des Bandes: Edith Nahler und Horst Nahler (1986); T. 4: Die Einzeldrucke. Bearbeiter des Bandes: Inge Jensen (1984).

Briefe an Goethe. Gesamtausgabe in Regestform $\langle R e-$ gestausgabe . Bd 1-5: 〈Im Auftrag der〉 Nationalen Forschungs- und Gedenkstätten der klassischen deutschen Literatur in Weimar. Goethe- und Schiller-Archiv hrsg. von Karl-Heinz Hahn. Redaktor: Irmtraut Schmid. Weimar 1980-1992; Ergänzungsband zu den Bänden 1-5. Hrsg. von der Stiftung Weimarer Klassik/Goethe- und Schiller-Archiv. Bearbeitet von Manfred Koltes unter Mitarbeit von Ulrike Bischof und Sabine Schäfer. Weimar 1995; Bd 6: Hrsg. von der Stiftung Weimarer Klassik/Goethe- und Schiller- 
Rackwitz, Collectaneen zu Reichardt

Reichardt-Goethe

Riemer

Roethe, Campagne in Frankreich
Archiv. Bearbeitet von Manfred Koltes unter Mitarbeit von Ulrike Bischof und Sabine Schäfer. 2 Tle. Weimar 2000; Bd 7: Hrsg. von der Stiftung Weimarer Klassik und Kunstsammlungen/Goethe- und Schiller-Archiv. Bearbeitet von Manfred Koltes unter Mitarbeit von Ulrike Bischof und Sabine Schäfer. 2 Tle. Weimar 2004; Bd 8: Hrsg. von der Klassik Stiftung Weimar/Goethe- und Schiller-Archiv. Bearbeitet von Manfred Koltes, Ulrike Bischof und Sabine Schäfer. 2 Tle. Weimar 2011; Bd 9: Hrsg. von der Klassik Stiftung Weimar/Goethe- und Schiller-Archiv. Bearbeitet von Manfred Koltes, Ulrike Bischof, Christoph Hain und Sabine Schäfer. 2 Tle. Weimar 2017.

Werner Rackwitz: „... Ich hätte Ihm weggejagt wen ich den Esel nicht über wichtigern Dingen vergeßen häte ...". Collectaneen zu Johann Friedrich Reichardts Lebensumständen in den Jahren 1790 bis 1795. In: Johann Friedrich Reichardt (1752-1814). Zwischen Anpassung und Provokation. Goethes Lieder und Singspiele in Reichardts Vertonung. Bericht über die wissenschaftlichen Konferenzen in Halle anlässlich des 250. Geburtstages 2002 und zum Goethejahr 1999. Hrsg. vom Händel-Haus Halle, vom Institut für Musikwissenschaft und vom Germanistischen Institut der Universität Halle durch Manfred Beetz, Kathrin Eberl, Konstanze Musketa und Wolfgang Ruf unter Mitarbeit von Katrin Keym, Götz Traxdorf und Jens Wehmann (Schriften des Händel-Hauses in Halle. Bd 19). Halle an der Saale 2003.

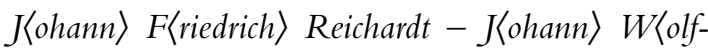
gang $\rangle$ Goethe. Briefwechsel. Hrsg. und kommentiert von Volkmar Braunbehrens, Gabriele Busch-Salmen, Walter Salmen. Weimar 2002.

Briefe von und an Goethe. Desgleichen Aphorismen und Brocardica. Hrsg. von Friedrich Wilhelm Riemer. Leipzig 1846.

Goethes Campagne in Frankreich 1792. Eine philologische Untersuchung aus dem Weltkriege von Gustav Roethe. Berlin 1919. 
Ruppert

Salmen, Reichardt

Satori-Neumann ${ }^{2}$

Schnauß, Lebens-Notizen

Schneider, Oper Berlin

SchrGG

Schulte-Strathaus

Sedlarz, Moritz'

Italienreise

Sigismund,

Prinz Constantin

Sömmerrings Leben
Goethes Sammlungen zu Kunst, Literatur und $\mathrm{Na}$ turwissenschaft. Hrsg. von den Nationalen Forschungs- und Gedenkstätten der klassischen deutschen Literatur in Weimar. Goethes Bibliothek. Katalog. Bearbeiter der Ausgabe Hans Ruppert. Weimar 1958.

Walter Salmen: Johann Friedrich Reichardt. Komponist, Schriftsteller, Kapellmeister und Verwaltungsbeamter der Goethezeit. Zweite erweiterte und ergänzte Auflage mit einer neuen Bibliographie. Hildesheim, Zürich, New York 2002.

Lothar Schirmer: Die Frühzeit des Weimarischen Hoftheaters unter Goethes Leitung (1791-1798). Nach den Quellen bearbeitet von Bruno Th〈omas $\rangle$ Satori-Neumann. Neu hrsg. und kommentiert. 2 Bde (Schriften der Gesellschaft für Theatergeschichte. Bd 80/1,2). Berlin 2013.

Christian Friedrich Schnauß: Lebens-Notizen. In: Carl von Beaulieu-Marconay: Ein Weimarer Beamter des achtzehnten Jahrhunderts. Zeitschrift für deutsche Kulturgeschichte. Neue Folge. IV. Jg. Hrsg. von Dr. J. H. Müller. Hannover 1875, S. 655-702.

L〈ouis〉 Schneider: Geschichte der Oper und des Königlichen Opernhauses in Berlin. Berlin 1852.

Schriften der Goethe-Gesellschaft.

Die Bildnisse Goethes. Hrsg. von Ernst SchulteStrathaus. München 1910 (Supplementband zur Propyläen-Ausgabe).

Claudia Sedlarz: Rom sehen und darüber reden. Karl Philipp Moritz' Italienreise 1786-1788 und die literarische Darstellung eines neuen Kunstdiskurses (Berliner Klassik. Eine Großstadtkultur um 1800. Studien und Dokumente, hrsg. von der Berlin-Brandenburgischen Akademie der Wissenschaften, betreut von Conrad Wiedemann. Bd 12). Hannover 2010. Volker L. Sigismund: Ein unbehauster Prinz - Constantin von Sachsen-Weimar (1758-1793), der Bruder des Herzogs Carl August. Ein biographischer Essay. In: GJb 106 (1989), 250-277.

Samuel Thomas Sömmerring's Leben und Verkehr mit seinen Zeitgenossen. Hrsg. von Rudolph Wagner. 
Soemmerring, Werke

Steiger

Stunden mit Goethe

Suphan

Theater in Berlin

Verzeichniß

Ostermesse 1792

Vierte Nachricht

Von und an Herder
Erste Abteilung: Briefe berühmter Zeitgenossen an Sömmerring. Leipzig 1844.

Samuel Thomas Soemmerring: Werke. Begründet von Gunter Mann. Hrsg. von Jost Benedum und Werner Friedrich Kümmel. 23 Bde. Stuttgart, Jena, Lübeck, Ulm 1990-1998 und Basel 1999-2011. Bd 20: Briefwechsel November 1792 - April 1805. Hrsg. und erläutert von Franz Dumont. Basel 2001. Goethes Leben von Tag zu Tag. Eine dokumentarische Chronik von Robert Steiger. 8 Bde (Bd 1-5 von Robert Steiger. Bd 6 von Robert Steiger und Angelika Reimann. Bd 7-8 von Angelika Reimann). Zürich, München 1982-1993 (Bd 1-6) und Zürich 19951996 (Bd 7-8). - Bd 3: 1789-1798 (1984). Generalregister. Hrsg. von Siegfried Seifert. Berlin, Boston 2011.

Stunden mit Goethe. Für die Freunde seiner Kunst und Weisheit. Hrsg. von Wilhelm Bode. 10 Bde. Berlin 1905-1921. - Bd 8 (1912).

Herders Sämmtliche Werke. Hrsg. von Bernhard Suphan. 33 Bde. Berlin 1877-1913.

Die Königlichen Theater in Berlin. Statistischer Rückblick auf die künstlerische Thätigkeit und die Personal-Verhältnisse während des Zeitraums vom 5. December 1786 bis 31. December 1885. Zusammengestellt von C. Schäffer und C. Hartmann. Berlin 1886.

Allgemeines Verzeichniß der Bücher, welche in der Frankfurter und Leipziger Ostermesse des 1792 Jahres entweder ganz neu gedruckt, oder sonst verbessert, wieder aufgeleget worden sind, auch inskünftige noch herauskommen sollen. Leipzig 1792.

Johann Wolfgang von Goethe, Christian Gottlob Voigt: Vierte Nachricht von dem Fortgang des neuen Bergbaues zu Ilmenau. Womit zugleich ein auf den Sechsten Junius 1791 zu eröffnender Gewerkentag ausgeschrieben wird. Weimar 1791.

Von und an Herder. Ungedruckte Briefe aus Herders Nachlaß. Hrsg. von Heinrich Düntzer und Ferdinand Gottfried von Herder. 3 Bde. Leipzig 18611862. - Bd 1: Herders Briefwechsel mit Gleim und 
Vulpius-Korrespondenz

$W A$

Wagner, Tagebuch

Wahle,

Weimarer Hoftheater

WAN

Wartenberg, Familienkreis Jacobi
Nicolai (1861); Bd 2: Herders Briefwechsel mit Hartknoch, Heyne und Eichhorn, Briefe an Grupen, Herders Gattin und J〈ohannes von〉 Müller, nebst Briefen von Fr〈iedrich $\rangle L\langle$ udwig $\rangle W\langle i l h e l m\rangle$ Meyer und $A\langle$ ugust $\rangle$ von Einsiedel (1861); Bd 3: Herders Briefwechsel mit Knebel, Karl von Dalberg, Joh〈ann〉 Friedr〈ich〉Hugo von Dalberg, einzelne Briefe an Herder, ungedruckte Gedichte und Uebersetzungen Herders, hodegetische Abendvorträge Herders, aus Briefen von Herders Gattin an J〈ohann $\rangle$ G $\langle$ eorg $\rangle$ Müller, Herders Antwort an den Kirchenconvent der Petersgemeine zu Petersburg (1862).

Christian August Vulpius: Eine Korrespondenz zur Kulturgeschichte der Goethezeit. Hrsg. von Andreas Meier. Bd 1: Brieftexte. Bd 2: Kommentar (Quellen und Forschungen zur Literatur- und Kulturgeschichte. Hrsg. von Ernst Osterkamp und Werner Röcke. Bd 28/1-2). Berlin, New York 2003.

Goethes Werke. Hrsg. im Auftrage der Großherzogin Sophie von Sachsen 〈Weimarer Ausgabe〉. 143 Bde.I. Abtheilung: Goethes Werke. 55 Bde. Weimar 1887-1918; II. Abtheilung: Goethes Naturwissenschaftliche Schriften. 13 Bde. Weimar 1890-1904; III. Abtheilung: Goethes Tagebücher. 15 Bde. Weimar 1887-1919; IV. Abtheilung: Goethes Briefe. 50 Bde. Weimar 1887-1912.

Johann Conrad Wagner: „Meine Erfahrungen in dem gegenwärtigen Kriege". Tagebuch des Feldzugs mit Herzog Carl August von Weimar. Hrsg. von Edith Zehm. Mit einer Einführung von Gustav Seibt (Schriften der Goethe-Gesellschaft 78). Göttingen 2018.

Das Weimarer Hoftheater unter Goethes Leitung. Aus neuen Quellen bearbeitet von Julius Wahle (SchrGG 6). Weimar 1892.

Goethes Werke. Weimarer Ausgabe. Nachträge und Register zur IV. Abteilung: Briefe. Hrsg. von Paul Raabe. 3 Bde. München 1990 (WA IV 51-53). Jan Wartenberg: Der Familienkreis Friedrich Heinrich Jacobi und Helene Elisabeth von Clermont. Bildnisse und Zengnisse. Hrsg. vom Goethe-Museum Düsseldorf Anton-und-Katharina-Kippenberg-Stif- 
$W B$

Wilson, Weimar und Revolution

Wingertszahn, Moritz-Vieweg

Zedler

Zehm, Frankreichfeldzug tung. Mit einem Geleitwort von Volkmar Hansen und einer Einführung von Gudrun Schury. Bonn 2011. Wielands Briefwechsel. 18 Bde. Berlin 1963-2005. Bd 1-2: Hrsg. von der Deutschen Akademie der Wissenschaften zu Berlin/Institut für deutsche Sprache und Literatur (Bd 2: durch Hans Werner Seiffert); Bd 3-5: Hrsg. von der Akademie der Wissenschaften der DDR/Zentralinstitut für Literaturgeschichte durch Hans Werner Seiffert; Bd 6-18: Hrsg. von der Berlin-Brandenburgischen Akademie der Wissenschaften durch Siegfried Scheibe. - Bd 10: April 1788-Dezember 1790. Erster T.: Text. Bearbeitet von Uta Motschmann (1992). Zweiter T.: Anmerkungen. Bearbeitet von Uta Motschmann (1993). Bd 11: Januar 1791 - Juni 1793. Erster T.: Text. Bearbeitet von Uta Motschmann (2001). Zweiter T.: Anmerkungen. Bearbeitet von Uta Motschmann (2003). Bd 12: Juli 1793 - Juni 1795. Erster T.: Text. Bearbeitet von Klaus Gerlach (1993). Zweiter T.: Anmerkungen. Bearbeitet von Klaus Gerlach (1995).

Goethes Weimar und die Französische Revolution. Dokumente der Krisenjahre. Hrsg. von W. Daniel Wilson. Köln, Weimar, Wien 2004.

Christoph Wingertszahn: „zu einer vorläufigen Ankündigung ist es immer genug". Unbekannte Mitteilungen von Karl Philipp Moritz an seinen Verleger Johann Friedrich Vieweg. In: Berliner Aufklärung. Kulturwissenschaftliche Studien. Bd 1. Hrsg. von Ursula Goldenbaum und Alexander Košenina. Hannover 1999, S. 220-230.

Grosses vollständiges Universal-Lexicon Aller Wissenschaften und Künste, Welche bishero durch menschlichen Verstand und Witz erfunden und verbessert worden. 〈...〉. Leipzig und Halle. Verlegts Johann Heinrich Zedler. 64 Bde. 1732-1750. 4 Supplementbde. Leipzig 1751-1754. - Bd 6: 1733; Bd 18: 1738; Bd 44: 1745.

Edith Zehm: Der Frankreichfeldzug von 1792. Formen seiner Literarisierung im Tagebuch Johann Conrad Wagners und in Goethes „Campagne in Frank- 
reich" (Europäische Hochschulschriften 1). Frankfurt a. M. u.a. 1985.

Zoeppritz, Jacobis Nachlaß Aus F. H. Jacobi's Nachlaß. Ungedruckte Briefe von und an Jacobi und Andere. Nebst ungedruckten Gedichten von Goethe und Lenz. Hrsg. von Rudolf Zoeppritz. 2 Bde. Leipzig 1869. 